\title{
On the global stable manifold
}

\author{
by \\ Alberto Abbondandolo and Pietro Majer (Pisa)
}

\begin{abstract}
We give an alternative proof of the stable manifold theorem as an application of the (right and left) inverse mapping theorem on a space of sequences. We investigate the diffeomorphism class of the global stable manifold, a problem which in the general Banach setting gives rise to subtle questions about the possibility of extending germs of diffeomorphisms.
\end{abstract}

Let $M$ be an open subset of a manifold $M^{\prime}$ of class $C^{k}, 1 \leq k \leq \omega$ (meaning that $k \in \mathbb{Z}^{+}$, or $k=\infty$, or $k=\omega$, where as usual $C^{\omega}$ denotes the analytic category) modeled on the Banach space $E$. Let $f: M \rightarrow f(M) \subset$ $M^{\prime}$ be a $C^{k}$ diffeomorphism $\left(^{1}\right)$, and let $x \in M$ be a hyperbolic fixed point of $f$. This means that the spectrum of $D f(x)$ does not meet the unit circle, thus it is divided into two disjoint closed subsets $\sigma(D f(x)) \cap\{|z|<1\}$ and $\sigma(D f(x)) \cap\{|z|>1\}$, and the spectral decomposition theorem gives a corresponding $D f(x)$-invariant decomposition of the tangent space of $M$ at $x, T_{x} M=E^{\mathrm{s}} \oplus E^{\mathrm{u}}$. The stable manifold of $x$ is the set

$$
W^{\mathrm{s}}(x)=\left\{p \in \bigcap_{n \in \mathbb{N}} f^{-n}(M) \mid \lim _{n \rightarrow \infty} f^{n}(p)=x\right\} .
$$

The stable manifold theorem states that $W^{\mathrm{s}}(x)$ is an immersed $C^{k}$ submanifold of $M$. A first way to prove such a result is to define the local stable manifold near $x$, to use the graph transform method to show that in local coordinates such a set is the graph of a Lipschitz map, then to prove further regularity, and finally to use the map $f$ to describe the whole stable manifold. See, for example, [Shu87]. Another approach is due to Irwin [Irw70], who replaces the graph transform method by an argument involving the implicit mapping theorem applied to the space of sequences (see

2000 Mathematics Subject Classification: 37D10, 46B20, 58B10.

Key words and phrases: stable manifold theorem, Banach manifold, hyperbolic fixed point, attractor.

$\left({ }^{1}\right)$ Usually $M^{\prime}=M=f(M)$, so that $f$ is a diffeomorphism of $M$. We allow this slightly more general setting to include the case of a local diffeomorphism. 
also [Wel76]). See [HPS77, Irw80, FHY83, Wig94, CFdlL03a, CFdlL03b] for many generalizations and for more bibliography.

The first aim of this note is to give a different proof of the stable manifold theorem. Denote by $c_{x}(M)$ the set of all $M$-valued sequences converging to $x$, and recall that $c_{x}(M)$ has a natural structure of $C^{k}$ manifold modeled on the Banach space $c_{0}(E)$, the space of infinitesimal $E$-valued sequences with the supremum norm (see Section 1). The stable manifold theorem can then be stated in the following form:

Theorem A. Let $M$ be an open subset of the $C^{k}$ Banach manifold $M^{\prime}$, let $f: M \rightarrow f(M) \subset M^{\prime}$ be a $C^{k}$ diffeomorphism, $1 \leq k \leq \omega$, and let $x \in M$ be a hyperbolic fixed point of $f$, inducing the $D f(x)$-invariant splitting $T_{x} M=E^{\mathrm{s}} \oplus E^{\mathrm{u}}$. Then the set

$$
\mathcal{W}=\left\{u \in c_{x}(M) \mid u(n+1)=f(u(n)) \forall n \in \mathbb{N}\right\}
$$

is a closed $C^{k}$ submanifold of $c_{x}(M)$, and the evaluation map at zero,

$$
\mathrm{ev}_{0}: c_{x}(M) \rightarrow M, \quad u \mapsto u(0),
$$

restricts to a $C^{k}$ injective immersion of $\mathcal{W}$ onto $W^{\mathrm{s}}(x)$ and has the property that $D \operatorname{ev}_{0}(x) T_{x} \mathcal{W}=E^{\mathrm{s}}$ (here $x$ denotes the constant sequence $\left.x(n)=x\right)$.

Therefore, $W^{\mathrm{s}}(x)$ is a $C^{k}$ immersed submanifold of $M$. Simple examples show that $W^{\mathrm{s}}(x)$ may not be an embedded submanifold, and may not even be locally closed. Lifting the dynamical system to the space of sequences produces instead the closed embedded submanifold $\mathcal{W}$. Notice also that $\left.\mathrm{ev}_{0}\right|_{\mathcal{W}}$ is a semi-conjugacy between the shift operator on $\mathcal{W}$ and the restriction of $f$ to $W^{\mathrm{s}}(x)$. By considering $f^{-1}$ instead of $f$ one finds an analogous statement for the unstable manifold $W^{\mathrm{u}}(x)$.

As in Irwin's approach, the proof of Theorem A uses the implicit mapping theorem on the space of sequences, but in a more direct way, due to the fact that we deal with zero sets of mappings instead of graphs. The whole analysis is reduced to quite a simple linear problem, and the regularity in the $C^{k}$ or even in the analytic case follows directly.

Let us sketch the proof of Theorem A in the case $M^{\prime}=M=\mathbb{R}^{n}$. Assuming that the fixed point $x$ is the origin, $\mathcal{W}$ is the zero set of the $C^{k}$ map

$$
F: c_{0}\left(\mathbb{R}^{n}\right) \rightarrow c_{0}\left(\mathbb{R}^{n}\right), \quad u \mapsto S u-f_{*}(u),
$$

where $S$ is the left shift $(S u)(n)=u(n+1)$, and $f_{*}$ denotes composition with $f$. It is readily seen that the set of $u \in \mathcal{W}$ such that $D F(u)$ has a right inverse is open and $f_{*}^{-1}$-invariant. Such a set contains the origin, because the linear mapping

$$
D F(0): c_{0}\left(\mathbb{R}^{n}\right) \rightarrow c_{0}\left(\mathbb{R}^{n}\right), \quad v \mapsto S v-D f(0)_{*} v,
$$


has a right inverse in the form of a convolution operator (see Lemma 2.1 below). The only $f_{*}^{-1}$-invariant neighborhood of 0 in $\mathcal{W}$ is the whole $\mathcal{W}$, so the implicit mapping theorem implies that $\mathcal{W}$ is a $C^{k}$ submanifold of $c_{0}\left(\mathbb{R}^{n}\right)$. The restriction to $\mathcal{W}$ of the map evo is clearly injective, and an argument similar to the one used above shows that it is an immersion.

The usual statement of the local stable manifold theorem is then deduced from Theorem A as a simple corollary (see Section 3). The same idea works for continuous-time dynamical systems, i.e. flows obtained by integrating some vector field on $M$ (see Remark 2.3).

Finally, we investigate the diffeomorphism class of the global stable manifold $W^{\mathrm{s}}(x)$ when $f$ is a global diffeomorphism of the Banach manifold $M$. In this case, it is natural to expect $\mathcal{W}$ to be $C^{k}$ diffeomorphic to the Banach space $E^{\mathrm{s}}$, so that the stable manifold is a $C^{k}$-immersed copy of $E^{\mathrm{s}}$. We do not know if this is true for manifolds $M$ modeled on an arbitrary Banach space. The difficulty in proving such a result is due to the fact that on Banach spaces the problem of extending the germ of a map by keeping the same regularity is quite delicate, because there need not exist a smooth norm, or smooth partitions of unity. We characterize those diffeomorphisms for which $\mathcal{W}$ is diffeomorphic to $E^{\mathrm{s}}$ in terms of an extension property for the germ of $f$ at $x$ (see Corollary 4.6), and we deduce the following result:

THEOREM B. Let $f$ be a $C^{k}$ diffeomorphism of the $C^{k}$ Banach manifold $M, 1 \leq k \leq \omega$, and let $x \in M$ be a hyperbolic fixed point of $f$, with associated splitting $T_{x} M=E^{\mathrm{s}} \oplus E^{\mathrm{u}}$. Then the manifold $\mathcal{W}$ is homeomorphic to the Banach space $E^{\mathrm{s}}$, by a bi-locally Lipschitz homeomorphism $\left({ }^{2}\right)$. Assume moreover that the Banach space $E^{\mathrm{S}}$ has the following property: there exists $a$ bounded $C^{k} \operatorname{map} \varphi: E^{\mathrm{s}} \rightarrow E^{\mathrm{s}}$ such that

(i) $\varphi$ coincides with the identity in a neighborhood of 0 , in the case $1 \leq k<\infty$

(ii) $\varphi(0)=0$ and $D \varphi(0)=I$, in the case $k=\infty$ or $k=\omega$.

Then $\mathcal{W}$ is $C^{k}$ diffeomorphic to the Banach space $E^{\mathrm{s}}$.

Therefore, $W^{\mathrm{s}}(x)$ is always the image of $E^{\mathrm{s}}$ under a locally Lipschitz and locally closed injective map, which can be chosen to be a $C^{k}$ immersion whenever the Banach space $E^{\mathrm{s}}$ has one of the properties described above.

Notice that these properties are hereditary, in the sense that if a Banach space $E$ admits a map $\varphi$ with one of these properties, then every comple-

$\left({ }^{2}\right)$ A map $h$ between Banach manifolds (on which there is no preferred metric) is said to be locally Lipschitz if $\varphi \circ h \circ \psi^{-1}$ is locally Lipschitz for every pair of local charts $\varphi$ and $\psi$. A homeomorphism $h$ is said to be bi-locally Lipschitz if both $h$ and $h^{-1}$ are locally Lipschitz. 
mented linear subspace of $E$ does (if the subspace $E^{\prime}$ is the image of the bounded linear projection $P$, consider $\left.\left.P \varphi\right|_{E^{\prime}}\right)$.

The property of admitting a $C^{k}$ bounded map coinciding with the identity in a neighborhood of 0 was introduced by Atkin [Atk01]. He observed that this property not only holds trivially when the Banach space $E$ admits a $C^{k}$ norm (so for instance when $E$ is a Hilbert space), but it also holds for some less regular Banach spaces such as $\ell_{\infty}$ or $C^{0}(K, \mathbb{R})$. This property implies that $C^{k}$ germs at $0 \in E$ have $C^{k}$ extensions to the whole Banach space $E$, a fact which is useful in order to make global constructions, also in the absence of $C^{k}$ partitions of unity.

Clearly, an Atkin map cannot exist in the analytic category, but in this case (and actually also in the smooth category) it is enough to assume the weaker condition (ii). Again, some non-regular Banach spaces, such as $\ell_{\infty}$ and $C^{0}(K, \mathbb{R})$, admit an analytic map satisfying (ii).

We do not know whether there exist Banach spaces which do not admit a $C^{k}$ map satisfying (i) or (ii).

Added in proof. Christopher Atkin recently informed us that he has a proof that every Banach space with a Schauder basis admits a bounded $C^{\infty}$ map satisfying (i).

\section{Notations, definitions and basic facts}

Linear operators and splittings. Let $\left(E,|\cdot|_{E}\right)$ and $\left(F,|\cdot|_{F}\right)$ be Banach spaces. We denote by $\mathcal{L}(E, F)$ the Banach space of all bounded linear operators from $E$ to $F$, endowed with the operator norm $\|T\|:=\sup _{|x|_{E} \leq 1}|T x|_{F}$. If $E=F$ we simply write $\mathcal{L}(E)$ for $\mathcal{L}(E, E)$. A linear subspace $X$ (necessarily closed) of $E$ is complemented in $E$ if and only if there exists a subspace $Y$ such that $E=X \oplus Y$. If $L \in \mathcal{L}(E, F)$ and $R \in \mathcal{L}(F, E)$ are such that $L R=I_{F}$, then $L$ is called a left inverse of $R$ or a linear retraction, and $R$ is called a right inverse of $L$ or a linear section. Then $L$ is surjective, $R$ is injective and $E$ decomposes as $E=\operatorname{ker} L \oplus \operatorname{ran} R$, with projections $P_{\text {ran } R}=R L$ and $P_{\text {ker } L}=I_{E}-R L$. Conversely, if $R \in \mathcal{L}(F, E)$ is injective and $E=X \oplus \operatorname{ran} R$ for some subspace $X$ of $E$, then $L:=R^{-1} P_{\operatorname{ran} R} \in \mathcal{L}(E, F)$ is a right inverse of $R$, with $\operatorname{ker} L=X$. Similarly, if $L \in \mathcal{L}(E, F)$ is surjective and $E=\operatorname{ker} L \oplus Y$ for some subspace $Y$ of $E$, then $R:=\left(L_{\mid Y}\right)^{-1}$ is a right inverse of $L$ with $\operatorname{ran} R=Y$. The set of linear sections $L \in \mathcal{L}(E, F)$ and the set of linear retractions $R \in \mathcal{L}(E, F)$ are open in $\mathcal{L}(E, F)$.

Immersions and submersions. Let $M, N$ be differentiable manifolds of class $C^{k}, 1 \leq k \leq \omega$, modeled on the Banach space $E$, respectively $F$. A map $f: M \rightarrow N$ is a local immersion (resp. a local submersion) at $p$ if $f$ is a linear section (resp. a linear retraction) in local charts at $p$, meaning that there exist a local chart at $p, \varphi: U \rightarrow \varphi(U) \subset E$, a local chart at $q:=f(p)$, $\psi: V \rightarrow \psi(V) \subset F$, and a linear operator $A \in \mathcal{L}(E, F)$ which is a linear 
section (resp. a linear retraction) such that $\psi f \varphi^{-1}=A_{\mid \varphi(U)}$. Then $Y:=$ $f(U)$ is a submanifold of $N$ and its tangent space at $q$ is $T_{q} Y=\operatorname{ran} D f(p)$ (resp. $X:=f^{-1}(q) \cap U$ is a submanifold of $M$ and its tangent space at $p$ is $T_{p} X=\operatorname{ker} D f(p)$ ). The map $f$ is said to be simply an immersion (resp. a submersion) if it is a local immersion (resp. a local submersion) at any $p \in M$. In the first case, if $f$ is also injective, $f(M)$ is said to be an immersed submanifold of $N$. If $f$ is a local submersion at every $p \in f^{-1}(q)$, then $f^{-1}(q)$ is an embedded submanifold of $M$.

The implicit mapping theorem implies the usual criterion for local immersions and submersions, stating that $f$ is a local immersion (resp. a local submersion) at $p$ if and only if $D f(p) \in \mathcal{L}\left(T_{p} M, T_{q} N\right)$ is a linear section (resp. a linear retraction). A standard reference is [Lan99, Section II, §2].

The criterion for local submersions has the following immediate consequence:

Proposition 1.1. Let $f, g: M \rightarrow N$ be $C^{k}$ maps between $C^{k}$ Banach manifolds, $1 \leq k \leq \omega$, and set

$$
W=\{p \in M \mid f(p)=g(p)\}
$$

If for every $p \in W$, the operator $D f(p)-D g(p) \in \mathcal{L}\left(T_{p} M, T_{f(p)} N\right)$ is a linear retraction, then $W$ is a $C^{k}$ submanifold of $M$, with $T_{p} W=\operatorname{ker}(D f(p)-$ $D g(p))$.

Indeed, the matter being local, we may assume that $N$ is an open subset of the Banach space $F$, so that $W$ is the zero set of the map $f-g$, which is by hypothesis a local submersion at every $p \in W$.

Discrete convolutions on $\ell_{p}$ classes. If $(E,|\cdot|)$ is a Banach space, the $\ell_{p}$-norm of $u: \mathbb{Z} \rightarrow E$ is $\|u\|_{p}:=\left(\sum_{n \in \mathbb{Z}}|u(n)|^{p}\right)^{1 / p}$ for $1 \leq p<\infty$, and $\|u\|_{\infty}:=\sup _{n \in \mathbb{Z}}|u(n)|$. Then $\ell_{p}(\mathbb{Z}, E)$ denotes the Banach space of all $u$ : $\mathbb{Z} \rightarrow E$ such that $\|u\|_{p}<\infty$. The set

$$
c_{0}(\mathbb{Z}, E):=\left\{u: \mathbb{Z} \rightarrow E \mid \lim _{|n| \rightarrow \infty} u(n)=0\right\}
$$

is a closed subspace of $\ell_{\infty}(\mathbb{Z}, E)$, and $\ell_{p}(\mathbb{Z}, E) \subset \ell_{q}(\mathbb{Z}, E) \subset c_{0}(\mathbb{Z}, E) \subset$ $\ell_{\infty}(\mathbb{Z}, E)$ for $1 \leq p \leq q<\infty$. The analogous class $\left\{u: \mathbb{N} \rightarrow E \mid \lim _{n \rightarrow \infty} u(n)\right.$ $=0\}$ is denoted simply by $c_{0}(E)$; it can be viewed as a closed complemented subspace of $c_{0}(\mathbb{Z}, E)$. Indeed, the identity mapping on $c_{0}(E)$ factors as $c_{0}(E) \stackrel{j}{\rightarrow} c_{0}(\mathbb{Z}, E) \stackrel{\varrho}{\rightarrow} c_{0}(E)$, the inclusion $j$ being given by zero-extension, and the map $\varrho$ by restriction to $\mathbb{N} \subset \mathbb{Z}$.

If $g \in \ell_{1}(\mathbb{Z}, \mathcal{L}(E))$ and $u \in \ell_{\infty}(\mathbb{Z}, E)$, their convolution product $g * u$ is defined by

$$
(g * u)(n):=\sum_{h \in \mathbb{Z}} g(n-h) u(h) .
$$


Young's inequality $\|g * u\|_{p} \leq\|g\|_{1}\|u\|_{p}$ implies that for any $p \in[1,+\infty]$ the convolution product is continuous as a bilinear map $\ell_{1}(\mathbb{Z}, \mathcal{L}(E)) \times \ell_{p}(\mathbb{Z}, E) \rightarrow$ $\ell_{p}(\mathbb{Z}, E)$. Furthermore, $g * u \in c_{0}(\mathbb{Z}, E)$ whenever $g \in \ell_{1}(\mathbb{Z}, \mathcal{L}(E))$ and $u \in$ $c_{0}(\mathbb{Z}, E)\left({ }^{3}\right)$.

Notice that the convolution with $g \in \ell_{1}(\mathbb{Z}, \mathcal{L}(E))$ defines a bounded linear operator $R_{g}$ on $c_{0}(E)$ by $u \mapsto g * u$ (more precisely, $R_{g} u=\varrho(g * j(u))$ ).

Manifolds of sequences. Let $M$ be a $C^{k}$ manifold modeled on the Banach space $E$, let $x \in M$, and let $c_{x}(M)$ be the set of sequences $u: \mathbb{N} \rightarrow M$ which converge to $x$. Equivalently, denoting by $\overline{\mathbb{N}}=\mathbb{N} \cup\{\infty\}$ the one-point compactification of the set of natural numbers, $c_{x}(M)$ is the set

$$
c_{x}(M)=\left\{u \in C^{0}(\overline{\mathbb{N}}, M) \mid u(\infty)=x\right\},
$$

so it can be endowed with the restriction of the compact-open topology of $C^{0}(\overline{\mathbb{N}}, M)$. The space $c_{x}(M)$ has the structure of a $C^{k}$ manifold modeled on the Banach space $c_{0}(E)$. Indeed, given $C^{k}$ local charts $\varphi_{n}: U_{n} \rightarrow \varphi_{n}\left(U_{n}\right)$ $\subset E, n=0, \ldots, m$, where $x \in U_{m}$ and $\varphi_{m}(x)=0$, consider the open subset of $c_{x}(M)$,

$$
\begin{aligned}
\mathcal{U} & =\mathcal{U}\left(U_{0}, \ldots, U_{m}\right) \\
& =\left\{u \in c_{x}(M) \mid u(n) \in U_{n} \forall n=0, \ldots, m-1, u(n) \in U_{m} \forall n \geq m\right\},
\end{aligned}
$$

and the homeomorphism $\Phi=\Phi\left(\varphi_{0}, \ldots, \varphi_{m}\right): \mathcal{U} \rightarrow \Phi(\mathcal{U}) \subset c_{0}(E)$ defined by

$$
\Phi(u)(n)= \begin{cases}\varphi_{n}(u(n)) & \text { if } 0 \leq n \leq m-1, \\ \varphi_{m}(u(n)) & \text { if } n \geq m\end{cases}
$$

It is easy to check that the collection of homeomorphisms $\Phi\left(\varphi_{0}, \ldots, \varphi_{m}\right)$ constitutes a $C^{k}$ atlas of $c_{x}(M)$. The tangent bundle of $c_{x}(M)$ is

$$
T c_{x}(M)=c_{0_{x}}(T M),
$$

where $0_{x}$ is the zero element of $T_{x} M \subset T M$, and its fibers are

$$
T_{u} c_{x}(M)=\left\{v: \mathbb{N} \rightarrow T M \mid v(n) \in T_{u(n)} M \forall n \in \mathbb{N}, \lim _{n \rightarrow \infty} v(n)=0_{x}\right\} .
$$

In particular, the tangent space of $c_{x}(M)$ at the constant sequence $x$ is $T_{x} c_{x}(M)=c_{0}\left(T_{x} M\right)$.

The (left) shift operator $\mathcal{S}: c_{x}(M) \rightarrow c_{x}(M), \mathcal{S}(u)(n)=u(n+1)$, is of class $C^{k}$, and its differential at $x$ is the (left) shift linear operator $S$ on $c_{0}\left(T_{x} M\right)$.

Also the evaluation at zero, $\mathrm{ev}_{0}: c_{x}(M) \rightarrow M, u \mapsto u(0)$, is a map of class $C^{k}$, and its differential at $u$ is the linear evaluation at zero, $D \operatorname{ev}_{0}(u)[v]=$ $v(0)$.

$\left({ }^{3}\right)$ This follows immediately by approximating $g$ with the sequence $g_{n}:=\mathbb{1}_{[-n, n]} g$, for $g_{n} \rightarrow g$ in $\ell_{1}, g_{n} * u \in c_{0}(\mathbb{Z}, E)$ and by Young's inequality $\left\|g * u-g_{n} * u\right\|_{\infty}=$ $\left\|\left(g-g_{n}\right) * u\right\|_{\infty} \leq\left\|g_{n}-g\right\|_{1}\|u\|_{\infty} \rightarrow 0$, so $g * u=\lim _{n \rightarrow \infty} g_{n} * u \in c_{0}(\mathbb{Z}, E)$. 
Finally, every continuous map $f: M \rightarrow N$ with $f(x)=y$ induces by composition a continuous map $f_{*}: c_{x}(M) \rightarrow c_{y}(N), f_{*}(u):=f \circ u$. If $f$ is of class $C^{k}, 1 \leq k \leq \omega$, so is $f_{*}$. Indeed, by taking local charts we are reduced to the case $M=E, N=F, x=y=0$, where the $h$ th differential of $f_{*}$ at $u \in c_{0}(E)$ is given by the formula

$$
\left(D^{h} f_{*}(u)[v]^{h}\right)(n)=D^{h} f(u(n))[v(n)]^{h} .
$$

In particular, the differential of $f_{*}$ at the constant sequence $x$ is the multiplication operator by $D f(x)$,

$$
\begin{gathered}
D f_{*}(x): T_{x} c_{x}(M)=c_{0}\left(T_{x} M\right) \rightarrow T_{y} c_{y}(N)=c_{0}\left(T_{y} N\right), \\
D f_{*}(x)[u](n)=D f(x)[u(n)] .
\end{gathered}
$$

Hyperbolic fixed points. An invertible operator $T \in \mathcal{L}(E)$ is said to be hyperbolic if its spectrum does not meet the unit circle: $\sigma(T) \cap\{|z|=1\}=\emptyset$. Then $\sigma(T)$ consists of the two disjoint closed subsets $\sigma(T) \cap\{|z|<1\}$ and $\sigma(T) \cap\{|z|>1\}$, so $E$ has the $T$-invariant spectral decomposition $E=$ $E^{\mathrm{s}} \oplus E^{\mathrm{u}}$, where $\sigma\left(\left.T\right|_{E^{\mathrm{s}}}\right)=\sigma(T) \cap\{|z|<1\}$ and $\sigma\left(\left.T\right|_{E^{\mathrm{u}}}\right)=\sigma(T) \cap\{|z|>1\}$.

A fixed point $x$ of a diffeomorphism $f: M \rightarrow f(M) \subset M^{\prime}$ is said to be hyperbolic if the differential of $f$ at $x, D f(x) \in \mathcal{L}\left(T_{x} M\right)$, is a hyperbolic operator. The corresponding spectral decomposition of the tangent space at $x$ is denoted by $T_{x} M=E^{\mathrm{s}} \oplus E^{\mathrm{u}}$.

2. Proof of the stable manifold theorem. Let us prove Theorem A. By definition,

$$
\mathcal{W}=\left\{u \in c_{x}(M) \mid \mathcal{S}(u)=f_{*}(u)\right\} .
$$

We start by studying the linear map $D \mathcal{S}(x)-D f_{*}(x) \in \mathcal{L}\left(T_{x} c_{x}(M)\right)$. By the discussion of Section 1, this is the linear operator

$$
S-D f(x)_{*}: c_{0}\left(T_{x} M\right) \rightarrow c_{0}\left(T_{x} M\right) .
$$

Let us simplify the notation by setting $E=T_{x} M, T=D f(x) \in \mathcal{L}(E)$. Denote by $P^{\mathrm{s}}$ and $P^{\mathrm{u}}$ the spectral projections associated to the decomposition $E=E^{\mathrm{s}} \oplus E^{\mathrm{u}}$ induced by the hyperbolic operator $T$.

Lemma 2.1. For $n \in \mathbb{Z}$, set

$$
g(n):=T^{n-1}\left(\mathbb{1}_{\mathbb{Z}^{+}}(n) I_{E}-P^{\mathrm{u}}\right),
$$

where $\mathbb{Z}^{+}=\{1,2, \ldots\}$, and $\mathbb{1}_{\mathbb{Z}^{+}}$is its characteristic function. Then $g \in$ $\ell_{1}(\mathbb{Z}, \mathcal{L}(E))$ and the corresponding convolution operator $R_{g} \in \mathcal{L}\left(c_{0}(E)\right)$ is a right inverse of $S-T_{*}$. Moreover,

$$
\operatorname{ker}\left(S-T_{*}\right)=\left\{u \in c_{0}(E) \mid u(n)=T^{n} u(0) \forall n \in \mathbb{N}, u(0) \in E^{\mathrm{s}}\right\} .
$$


Proof. Let $\|\cdot\|$ be the operator norm induced by a Banach norm on $E$. By the spectral radius theorem

$$
\begin{gathered}
\lim _{n \rightarrow \infty}\|g(n)\|^{1 / n}=\lim _{n \rightarrow \infty}\left\|T^{n-1} P^{\mathrm{s}}\right\|^{1 / n}=\lim _{n \rightarrow \infty}\left\|\left.T\right|_{E^{\mathrm{s}}} ^{n-1}\right\|^{1 / n}=\max \left|\sigma\left(\left.T\right|_{E^{\mathrm{s}}}\right)\right|<1, \\
\lim _{n \rightarrow \infty}\|g(-n)\|^{1 / n}=\lim _{n \rightarrow \infty}\left\|T^{-(n+1)} P^{\mathrm{u}}\right\|^{1 / n}=\lim _{n \rightarrow \infty}\left\|\left.T\right|_{E^{\mathrm{u}}} ^{-(n+1)}\right\|^{1 / n} \\
=\max \left|\sigma\left(\left.T\right|_{E^{\mathrm{u}}} ^{-1}\right)\right|<1 .
\end{gathered}
$$

Therefore, $g(n)$ tends to 0 exponentially fast for $|n| \rightarrow \infty$, in particular $g$ is in $\ell_{1}(\mathbb{Z}, \mathcal{L}(E))$.

For any $u \in c_{0}(E)$ and $n \in \mathbb{N}$, we have

$$
\begin{aligned}
{\left[\left(S-T_{*}\right) R_{g}(u)\right](n) } & =\sum_{h=0}^{\infty} g(n+1-h) u(h)-\sum_{h=0}^{\infty} T g(n-h) u(h) \\
& =\sum_{h=0}^{\infty} T^{n-h}\left[\mathbb{1}_{\mathbb{Z}^{+}}(n+1-h)-\mathbb{1}_{\mathbb{Z}^{+}}(n-h)\right] u(h) \\
& =\sum_{h=0}^{\infty} T^{n-h_{1}} \mathbb{1}_{\{0\}}(n-h) u(h)=u(n),
\end{aligned}
$$

that is, $\left(S-T_{*}\right) R_{g}=I_{c_{0}(E)}\left({ }^{4}\right)$. Finally, it is clear that $u \in \operatorname{ker}\left(S-T_{*}\right)$ if and only if $u(n)=T^{n} u(0)$ for any $n \in \mathbb{N}$, which defines an element of $c_{0}(E)$ if and only if $u(0) \in E^{\mathrm{s}}$.

Let us prove that the closed subset $\mathcal{W}$ is a $C^{k}$ submanifold of $c_{x}(M)$. By Lemma 2.1, DS $(x)-D f_{*}(x)$ is a linear retraction. Since the space of linear retractions is open, $D \mathcal{S}(u)-D f_{*}(u)$ is a linear retraction for every $u \in \mathcal{W} \cap U$, for a suitable neighborhood $U$ of $x$ in $c_{x}(M)$.

On the other hand, $f_{*}$ and $\mathcal{S}$ commute. As a consequence, if $u \in \mathcal{W}$ then $u_{n}=f_{*}^{n}(u)$ is also in $\mathcal{W}$, and the linear operator $D \mathcal{S}(u)-D f_{*}(u)$ is obtained from $D \mathcal{S}\left(u_{n}\right)-D f_{*}\left(u_{n}\right)$ by left and right multiplication by invertible linear operators. Since $u_{n}$ eventually belongs to $\mathcal{W} \cap U, D \mathcal{S}\left(u_{n}\right)-D f_{*}\left(u_{n}\right)$ is a linear retraction, and so is $D \mathcal{S}(u)-D f_{*}(u)$. Therefore, Proposition 1.1

$\left({ }^{4}\right)$ Here is a more heuristic argument to find a right inverse for the linear operator $S-T_{*}$. First notice that the equation $\left(S-T_{*}\right) u=w \in c_{0}(E)$ is equivalent to $u(n+1)=$ $T u(n)+w(n), \forall n \geq 0$. Iterating this gives $u(n)=T^{n} u(0)+\sum_{h=0}^{n-1} T^{n-1-h} w(h)$. We can split this equation into $u(n)=T^{n} P^{\mathrm{s}} u(0)+\sum_{h=0}^{n-1} T^{n-1-h} P^{\mathrm{s}} w(h)+T^{n}\left[P^{\mathrm{u}} u(0)+\right.$ $\left.\sum_{h=0}^{n-1} T^{-1-h} P^{\mathrm{u}} w(h)\right]$. Now the first and second terms converge as $n \rightarrow \infty$, because the spectral radius theorem implies that $\left\|T^{n} P^{\mathrm{s}}\right\| \leq c \lambda^{n}$ for some $c \geq 1$ and $\lambda<1$. The third term may not converge unless the sequence in the square brackets converges to 0 , that is, $P^{\mathrm{u}} u(0)+\sum_{h=0}^{n-1} T^{-1-h} P^{\mathrm{u}} w(h)=-\sum_{h=n}^{\infty} T^{-1-h} P^{\mathrm{u}} w(h)$, whence $u(n)=T^{n} P^{\mathrm{s}} u(0)+$ $(g * w)(n)$. 
implies that $\mathcal{W}$ is a $C^{k}$ submanifold of $c_{x}(M)$, with

$$
\begin{aligned}
T_{x} \mathcal{W} & =\operatorname{ker}\left(D \mathcal{S}(x)-D f_{*}(x)\right) \\
& =\left\{v \in c_{0}\left(T_{x} M\right) \mid v(n)=D f(x)^{n} v(0) \forall n \in \mathbb{N}, v(0) \in E^{\mathrm{S}}\right\} .
\end{aligned}
$$

The $C^{k}$ map ev $: c_{x}(M) \rightarrow M$ is clearly injective on $\mathcal{W}$, and it remains to show that for any $u \in \mathcal{W}, D \operatorname{ev}_{0} \mid \mathcal{W}(u): T_{u} \mathcal{W} \rightarrow T_{u(0)} M$ is a linear section. This is clearly true if $u=x$, for (1) shows that $D$ ev $_{0}(x)$ is an isomorphism onto $E^{\mathrm{s}}$, which is complemented in $T_{x} M$. Since the space of linear sections is open, the same is true for every $u$ in a neighborhood of $x$ in $\mathcal{W}$. The formula $\mathrm{ev}_{0} \circ f_{*}=f \circ \mathrm{ev}_{0}$ implies that $\left.D \mathrm{ev}_{0}(u)\right|_{T_{u} \mathcal{W}}$ is obtained from $\left.D \mathrm{ev}_{0}\left(u_{n}\right)\right|_{T_{u_{n}} \mathcal{W}}$ by left and right multiplication by invertible operators. As before, since $u_{n}$ converges to $x$, we conclude that $\left.D \mathrm{ev}_{0}(u)\right|_{T_{u} \mathcal{W}}$ is a linear section for every $u \in \mathcal{W}$. The proof of Theorem A is complete.

REMARK 2.2. The fact that $D \mathcal{S}(u)-D f_{*}(u)$ is a linear retraction can also be proved directly by the following generalization of Lemma 2.1: if $\mathcal{T}: c_{0}(E) \rightarrow c_{0}(E)$ is the multiplication operator by a sequence $\left(T_{n}\right) \subset \mathcal{L}(E)$ converging to a hyperbolic operator, then $S-\mathcal{T} \in \mathcal{L}\left(c_{0}(E)\right)$ is a linear retraction.

REMARK 2.3. A similar argument yields the stable manifold theorem for a hyperbolic equilibrium point $x$ of the local flow determined by a $C^{k}$ vector field $X$ on a $C^{k+1}$ Banach manifold $M$, where $1 \leq k \leq \omega$. Denote by $C_{x}^{1}\left(\left[0,+\infty[, M)\right.\right.$ the space of $C^{1}$ curves $[0,+\infty[\rightarrow M$ converging to $x$ as $t \rightarrow+\infty$, with the first derivative converging to 0 . Then one can use the implicit function theorem to prove that the set

$$
\mathcal{W}=\left\{u \in C _ { x } ^ { 1 } \left(\left[0,+\infty[, M) \mid u^{\prime}(t)-X(u(t))=0\right\}\right.\right.
$$

is a $C^{k}$ submanifold of $C_{x}^{1}([0,+\infty[, M)$, and that the evaluation at 0 restricts to a $C^{k}$ immersion of $\mathcal{W}$ onto the stable manifold of 0 . The basic linear step consists in proving that if $L \in \mathcal{L}(E)$ is infinitesimally hyperbolic (i.e. its spectrum does not meet the imaginary axis), then the operator

$$
\frac{d}{d t}-L: C_{0}^{1}\left(\left[0,+\infty[, E) \rightarrow C_{0}^{0}([0,+\infty[, E)\right.\right.
$$

is a linear retraction. See [AM04] for more details.

\section{The local stable manifold theorem}

Adapted norms. An adapted norm for a hyperbolic operator $T \in \mathcal{L}(E)$ is an equivalent norm $|\cdot|$ on $E$ such that

$$
\begin{aligned}
& |\xi|=\max \left\{\left|P^{\mathrm{s}} \xi\right|,\left|P^{\mathrm{u}} \xi\right|\right\}, \quad|T \xi| \leq \lambda|\xi| \quad \forall \xi \in E^{\mathrm{s}}, \\
& \left|T^{-1} \xi\right| \leq \lambda|\xi| \quad \forall \xi \in E^{\mathrm{u}},
\end{aligned}
$$


for some $0<\lambda<1$. One can always find a norm with these properties, provided that $\lambda>\max |\sigma(T) \cap\{|z|<1\}|$ and $\lambda>1 / \min |\sigma(T) \cap\{|z|>1\}|$. Indeed, the following stronger statement holds: if the spectrum of $T$ is contained in the annulus $\{\alpha<|z|<\beta\}$, then $E$ has an equivalent norm $|\cdot|$ such that, denoting by $\|\cdot\|$ the corresponding operator norm,

$$
\|T\| \leq \beta, \quad\left\|T^{-1}\right\| \leq 1 / \alpha .
$$

If $|\cdot|_{0}$ is any equivalent norm on $E$, a norm $|\cdot|$ satisfying (3) can be defined as

$$
|\xi|=\sum_{n=1}^{\infty} \alpha^{n}\left|T^{-n} \xi\right|_{0}+\sum_{n=0}^{\infty} \beta^{-n}\left|T^{n} \xi\right|_{0},
$$

as shown by the spectral radius theorem (see for instance [HPS77, Proposition 2.8]).

The local stable manifold. Let $U$ be an open neighborhood of 0 in the Banach space $E$, and let $f: U \rightarrow f(U) \subset E$ be a $C^{k}$ diffeomorphism, $1 \leq k \leq \omega$, having 0 as a hyperbolic fixed point. Let $T=D f(0)$, let $E=E^{\mathrm{s}} \oplus E^{\mathrm{u}}$ be the corresponding splitting, and let $|\cdot|$ be an adapted norm on $E$ for the hyperbolic operator $T$. If $V$ is a closed linear subspace of $E$, we denote by $V(r)$ the closed ball in $V$ of radius $r$. By the first property of adapted norms $(2), E(r)=E^{\mathrm{s}}(r) \times E^{\mathrm{u}}(r)$.

Given $r>0$ such that $E(r) \subset U$, the local stable manifold of 0 is the set

$$
W_{\mathrm{loc}, r}^{\mathrm{s}}(0):=\left\{p \in \bigcap_{n \in \mathbb{N}} f^{-n}(E(r)) \mid \lim _{n \rightarrow \infty} f^{n}(p)=0\right\} .
$$

This definition depends on $r$. However, if $r_{0}$ is small enough,

$$
W_{\mathrm{loc}, r}^{\mathrm{s}}(0)=W_{\mathrm{loc}, r_{0}}^{\mathrm{s}}(0) \cap E(r) \quad \forall r \leq r_{0} .
$$

Indeed, the first set is contained in the second by definition. Let us prove that the other inclusion holds when $r_{0}$ is small. The point $0 \in \mathcal{W}$ is a fixed point of the $C^{k}$ map $\left.f_{*}\right|_{\mathcal{W}}: \mathcal{W} \rightarrow \mathcal{W}$. By (1) and by the second property of adapted norms (2),

$$
\left\|\left.D f_{*}(0)\right|_{T_{0} \mathcal{W}}\right\|=\left\|\left.T_{*}\right|_{T_{0}} \mathcal{W}\right\| \leq \lambda<1,
$$

so a first order approximation shows that $f_{*} \mid \mathcal{W}$ is locally a contraction at 0 . In particular, there exists $r_{0}>0$ such that $\left\|f_{*}(u)\right\|_{\infty} \leq\|u\|_{\infty}$ for every $u \in \mathcal{W}$ with $\|u\|_{\infty} \leq r_{0}$. Since $f_{*}$ coincides with the shift $S$ on $\mathcal{W}$, this is equivalent to saying that $|u(n)|=\left|f^{n}(u(0))\right|$ is a decreasing sequence if $u \in \mathcal{W}$ and $\|u\|_{\infty} \leq r_{0}$. The conclusion follows.

THEOREM 3.1 (Local stable manifold theorem). Let $U$ be an open neighborhood of 0 in the Banach space $E, f: U \rightarrow f(U) \subset E$ be a $C^{k}$ diffeomorphism, $1 \leq k \leq \omega$, having 0 as a hyperbolic fixed point, inducing the splitting 
$E=E^{\mathrm{s}} \oplus E^{\mathrm{u}}$. If $r>0$ is small enough, then $W_{\mathrm{loc}, r}^{\mathrm{s}}(0)$ is the graph of a $C^{k}$ map $w: E^{\mathrm{S}}(r) \rightarrow E^{\mathrm{u}}(r)$ such that $w(0)=0$ and $D w(0)=0$.

Proof. Since $\mathrm{ev}_{0} \mid \mathcal{W}$ is a $C^{k}$ immersion, it is an embedding of an open neighborhood of 0 in $\mathcal{W}$. Therefore, if $r_{0}$ is small enough the set

$$
\left\{\operatorname{ev}_{0}(u) \mid u \in \mathcal{W},\|u\|_{\infty}<r_{0}\right\}
$$

is a $C^{k}$ submanifold of $E$, with tangent space at 0 equal to $D \operatorname{ev}_{0}(0) T_{0} \mathcal{W}=$ $E^{\mathrm{s}}$. Then, if $r<r_{0}$ is small enough, the set

$$
W_{\text {loc }, r_{0}}^{\mathrm{s}}(0) \cap E(r)=\left\{\operatorname{ev}_{0}(u) \mid u \in \mathcal{W},\|u\|_{\infty}<r_{0}\right\} \cap E(r)
$$

is the graph of a $C^{k}$ map $w: E^{\mathrm{s}}(r) \rightarrow E^{\mathrm{u}}(r)$ such that $w(0)=0$ and $D w(0)=0$, and the conclusion follows from (4).

\section{The diffeomorphism class of the global stable manifold}

Characterization of diffeomorphisms such that $W^{\mathrm{s}}(x)$ is an immersed copy of $E^{\mathrm{s}}$. A hyperbolic fixed point $x$ of a diffeomorphism $f: M \subset M^{\prime} \rightarrow$ $f(M) \subset M^{\prime}$ is said to be a local attractor if $W^{\mathrm{s}}(x)$ is a neighborhood of $x$, or equivalently ( $\left.{ }^{5}\right)$ if $E^{\mathrm{s}}=T_{x} M$. It is said to be a global attractor if $W^{\mathrm{s}}(x)=M$ (in particular, $f(M) \subset M$ ), in which case $f$ is said to be a topological contraction of $M$.

DeFinition 4.1. Let $U$ be an open neighborhood of 0 in the Banach space $E$, and let $f: U \rightarrow f(U) \subset E$ be a diffeomorphism of class $C^{k}$, $1 \leq k \leq \omega$, such that the hyperbolic fixed point 0 is a local attractor. We say that the germ of $f$ at 0 extends to a $C^{k}$ topological contraction of $E$ if it can be represented by a global $C^{k}$ diffeomorphism of $E$ having 0 as a global attractor.

In other words, we require the existence of a global diffeomorphism $\tilde{f}$ : $E \rightarrow E$ of class $C^{k}$ which coincides with $f$ in a neighborhood $V \subset U$ of 0 , and which is a topological contraction. If we want $\widetilde{f}$ to be only Lipschitz, its existence is always guaranteed:

LEMMA 4.2. Under the assumptions of Definition 4.1, there always exists a homeomorphism $\widetilde{f}: E \rightarrow E$ such that $\widetilde{f}$ and $\widetilde{f}^{-1}$ are globally Lipschitz, $\widetilde{f}$ coincides with $f$ in a neighborhood $V \subset U$ of 0 , and 0 is a global attractor.

Proof. Let $T=D f(0)$ and let $|\cdot|$ be an adapted norm for $T$; if $\|\cdot\|$ denotes the corresponding operator norm, we have $\|T\|<1$. Let $\varphi: E \rightarrow E$ be a $k$ Lipschitz bounded map which coincides with the identity in a neighborhood of 0 (for instance, $\varphi(\xi)=\chi(|\xi|) \xi$ with $\chi(s)=1$ for $s \leq 1, \chi(s)=2-s$ for $1 \leq s \leq 2, \chi(s)=0$ for $s \geq 2$, has the required properties, with $\operatorname{lip} \varphi \leq 3)$.

$\left({ }^{5}\right)$ Indeed, if $E^{\mathbf{s}} \neq T_{x} M$ the local stable manifold has empty interior, so by the Baire theorem the global stable manifold cannot fill an open set. 
Write $f$ as $f(\xi)=T\left(\xi+f_{0}(\xi)\right)$. Since $f_{0}$ is at least $C^{1}$ and $D f_{0}(0)=0$, we can find a neighborhood $U_{0}$ of 0 such that

$$
\left.\operatorname{lip} f_{0}\right|_{U_{0}}<1 / k, \quad \theta:=\left(1+\left.k \operatorname{lip} f_{0}\right|_{U_{0}}\right)\|T\|<1 .
$$

Up to replacing $\varphi(\xi)$ by $\lambda \varphi(\xi / \lambda)$ — which is still $k$-Lipschitz - we may assume that $\varphi(E) \subset U_{0}$, and we set

$$
\tilde{f}: E \rightarrow E, \quad \widetilde{f}(\xi)=T\left(\xi+f_{0}(\varphi(\xi))\right) .
$$

The map $\tilde{f}$ is a Lipschitz diffeomorphism of $E$ onto $\underset{\sim}{E}$ together with its inverse because $\operatorname{lip}\left(f_{0} \circ \varphi\right) \leq\left. k \operatorname{lip} f_{0}\right|_{U_{0}}<1$. Moreover, $\widetilde{f}=f$ in a neighborhood of 0 , and

$$
|\widetilde{f}(\xi)| \leq\|T\|\left(|\xi|+\left|f_{0}(\varphi(\xi))\right|\right) \leq\|T\|\left(1+\left.k \operatorname{lip} f_{0}\right|_{U_{0}}\right)|\xi|=\theta|\xi|,
$$

so the fact that $\theta<1$ implies that 0 is a global attractor.

In the analytic category the extension of a germ is unique (and not always possible, even in finite-dimensional spaces), so the requirement of Definition 4.1 is quite strong. A weaker property is given by the following:

DEFinition 4.3. Under the same assumptions of Definition 4.1, we say that the germ of $f$ at 0 extends up to conjugacy to a $C^{k}$ topological contraction of $E$ if there exists a $C^{k}$ diffeomorphism $h: V \rightarrow h(V)$ of an open neighborhood of 0 with $h(0)=0$ such that the germ of $h \circ f \circ h^{-1}$ at 0 extends to a topological contraction of $E$.

For instance, if an analytic diffeomorphism is analytically linearizable near a hyperbolic local attractor, then it extends up to conjugacy to an analytic topological contraction of $E$. This latter definition is relevant also in the smooth and in the finite differentiability category because it extends to diffeomorphisms defined on manifolds:

Definition 4.4. If $U$ is an open neighborhood of $x$ in the Banach manifold $M$ (modeled on the Banach space $E$ ) and $f: U \rightarrow f(U) \subset M$ is a $C^{k}$ diffeomorphism, $1 \leq k \leq \omega$, having $x$ as a local attractor, we say that the germ of $f$ at $x$ extends up to conjugacy to a $C^{k}$ topological contraction of $E$ if the germ of diffeomorphism on an open neighborhood of 0 in $E$ defined by conjugacy by a local chart (mapping $x$ into 0 ) extends up to conjugacy to a topological contraction of $E$.

Clearly, this definition does not depend on the choice of the local chart.

THEOREM 4.5. Let $f$ be a $C^{k}$ diffeomorphism of the $C^{k}$ Banach manifold $M$ modeled on the Banach space $E, 1 \leq k \leq \omega$, and assume that the hyperbolic fixed point $x \in M$ is a global attractor. Then $M$ is homeomorphic to $E$ by a bi-locally Lipschitz homeomorphism. Furthermore, $M$ is $C^{k}$ diffeomorphic to $E$ if and only if the germ of $f$ at $x$ extends up to conjugacy to a $C^{k}$ topological contraction of $E$. 
Proof. Let $U \subset M$ be an open neighborhood of $x$ in $M$, and let $\psi$ : $U \rightarrow \psi(U) \subset E$ be a $C^{k}$ local chart with $\psi(x)=0$. Consider the $C^{k}$ diffeomorphism

$$
g=\psi \circ f \circ \psi^{-1}: \psi\left(U \cap f^{-1}(U)\right) \rightarrow \psi(U \cap f(U)) \subset E .
$$

By Lemma 4.2, there exists an invertible topological contraction $h: E \rightarrow$ $E$ which is locally Lipschitz together with its inverse and which coincides with $g$ in an open neighborhood $V \subset \psi\left(U \cap f^{-1}(U)\right)$ of 0 . If we are also assuming that the germ of $f$ at $x$ extends up to conjugacy to a $C^{k}$ topological contraction of $E$, up to changing the local chart $\psi$ we may assume that $h$ is a $C^{k}$ diffeomorphism.

Let us define the global homeomorphism $\phi: M \rightarrow E$. Let $p \in M$. Since $W^{\mathrm{s}}(x)=M$, there exists $n \in \mathbb{N}$ such that $f^{n}(p) \in \psi^{-1}(V)$, and we set

$$
\phi(p)=h^{-n}\left(\psi\left(f^{n}(p)\right)\right) .
$$

Since $\psi$ conjugates the diffeomorphisms $\left.f\right|_{\psi^{-1}(V)}$ and $\left.h\right|_{V}=\left.g\right|_{V}$, this definition does not depend on the choice of $n$. The map $\phi$ is invertible, its inverse being the map

$$
\phi^{-1}(\xi)=f^{-n}\left(\psi^{-1}\left(h^{n}(\xi)\right)\right),
$$

for $n=n(p)$ so large that $h^{n}(\xi) \in V$. So $\phi$ is the required locally Lipschitz homeomorphism, and when $h$ is a $C^{k}$ diffeomorphism, $\phi$ is also a $C^{k}$ diffeomorphism.

Conversely, assume that there is a $C^{k}$ diffeomorphism $\phi: M \rightarrow E$. Up to composition with a translation, we may assume that $\phi(x)=0$. In particular, $\phi$ is a local chart mapping $x$ into 0 , and $\phi \circ f \circ \phi^{-1}$ is a global diffeomorphism of $E$ onto $E$ which is a topological contraction of $E$. This shows that the germ of $f$ at $x$ extends up to conjugacy to a $C^{k}$ topological contraction of $E$.

Let us consider the general case $W^{\mathrm{s}}(x) \neq M$. Denote by $W_{\mathrm{loc}}^{\mathrm{s}}(x)$ the image under $\mathrm{ev}_{0}$ of a neighborhood of $x$ in $\mathcal{W}$ so small that ev $\mathrm{v}_{0}$ is an embedding on it. Then $W_{\text {loc }}^{\mathrm{s}}(x) \subset W^{\mathrm{s}}(x)$ is a $C^{k}$ submanifold modeled on the Banach space $E^{\mathrm{s}}$.

Corollary 4.6. Let $f: M \rightarrow M$ be a $C^{k}$ diffeomorphism of a Banach manifold, $1 \leq k \leq \omega$. Let $x$ be a hyperbolic fixed point of $f$, with associated splitting $T_{x} M=E^{\mathrm{s}} \oplus E^{\mathrm{u}}$. Then the $C^{k}$ manifold $\mathcal{W}$ is homeomorphic to $E^{\mathrm{s}}$ by a bi-locally Lipschitz homeomorphism. Furthermore, $\mathcal{W}$ is $C^{k}$ diffeomorphic to $E^{\mathrm{s}}$ if and only if the germ of $\left.f\right|_{W_{\mathrm{loc}}^{\mathrm{s}}(x)}$ at $x$ extends up to conjugacy to a $C^{k}$ topological contraction of $E^{\mathrm{s}}$.

Indeed, it is enough to apply Theorem 4.5 to the diffeomorphism $f_{*}$ of the manifold $\mathcal{W}$. 
Since the evaluation at zero defines a local conjugacy between the restriction of $f_{*}$ to a small neighborhood of $x$ in $\mathcal{W}$ and the restriction of $f$ to $W_{\text {loc }}^{\mathrm{s}}(x)$, the above corollary can be restated in the following way: $W^{\mathrm{s}}(x)$ is always the image of a locally Lipschitz and locally closed injective map $E^{\mathrm{s}} \hookrightarrow M$, which can be chosen to be also a $C^{k}$ immersion if and only if the germ of $\left.f\right|_{W_{\text {loc }}^{\mathrm{s}}(x)}$ at $x$ extends up to conjugacy to a $C^{k}$ topological contraction of $E^{\mathrm{s}}$.

The statement about the locally Lipschitz homeomorphism in the above corollary is the first part of Theorem B.

Regularity conditions on the Banach space $E$. If $1 \leq k \leq \infty$ and the Banach space is somehow regular, one may expect that every germ of $C^{k}$ diffeomorphism having 0 as a hyperbolic local attractor extends to a $C^{k}$ topological contraction of $E$. This is actually true for a large class of Banach spaces. Indeed let us consider the following conditions on $E$ :

(E1) $E$ is finite-dimensional;

(E2) $E$ has a Hilbert structure;

(E3) there exists a $C^{k}$ norm on $E$ (i.e. an equivalent norm $|\cdot|$ such that the function $\xi \mapsto|\xi|$ is $C^{k}$ on $\left.E \backslash\{0\}\right)$;

(E4) there exists a bounded $C^{k}$ map $\varphi: E \rightarrow E$ which coincides with the identity on a neighborhood of 0 ;

(E5) there exists a bounded $C^{k} \operatorname{map} \varphi: E \rightarrow E$ such that $\varphi(0)=0$ and $D \varphi(0)=I$.

It is readily seen that $(\mathrm{E} 1) \Rightarrow(\mathrm{E} 2) \Rightarrow(\mathrm{E} 3) \Rightarrow(\mathrm{E} 4) \Rightarrow(\mathrm{E} 5)$. For instance, if $E$ admits a $C^{k}$ norm $|\cdot|$, a $C^{k}$ map $\varphi$ satisfying (E4) can be defined as

$$
\varphi(\xi)=\chi(|\xi|) \xi
$$

where $\chi:[0,+\infty) \rightarrow \mathbb{R}$ is a smooth function with compact support and such that $\chi(s)=s$ for $s \leq 1$. Condition (E4) was considered by Atkin [Atk01], who observed that it may also hold for Banach spaces which do not have regular norms. For instance, the Banach space $\ell_{\infty}=\ell_{\infty}(\mathbb{N}, \mathbb{R})$ does not admit a smooth norm, but it supports a smooth map $\varphi$ satisfying $(\mathrm{E} 4)$, namely

$$
\varphi(u)(n)=\chi(u(n)), \quad \forall n \in \mathbb{N},
$$

where $\chi: \mathbb{R} \rightarrow \mathbb{R}$ is a smooth bounded function coinciding with the identity in a neighborhood of 0 . A similar construction works for the Banach space $L^{\infty}(X, \mathcal{F}, \mu),(X, \mathcal{F}, \mu)$ a measure space, and for the $\operatorname{Banach}$ space $C^{0}(K, \mathbb{R})$, $K$ a compact topological space.

Condition (E5) holds for a large class of Banach spaces, even in the case $k=\omega$, when (E4) is obviously never fulfilled. For instance, the spaces $L^{\infty}(X, \mathcal{F}, \mu)$ and $C^{0}(K, \mathbb{R})$ admit the analytic map

$$
u \mapsto \sin u
$$


which satisfies (E5). It is not clear whether (E4) and (E5) are equivalent for $k<\omega$.

Even if we do not know any counterexample, it is not likely that every Banach space admits a smooth map satisfying (E4) or (E5). In any case, it would be interesting to characterize the class of Banach spaces which admit such maps. At the moment, the situation is unclear even for simple spaces such as $\ell^{1}$.

The case of finite differentiability. The following result, together with Corollary 4.6, implies part (i) of Theorem B.

Proposition 4.7. Let $1 \leq k \leq \infty$, and assume that the Banach space $E$ satisfies condition (E4) above. Let $U \subset E$ be an open neighborhood of 0 , and let $f: U \rightarrow f(U) \subset E$ be a $C^{k}$ diffeomorphism with hyperbolic fixed point 0 which is a local attractor. Then the germ of $f$ at 0 extends to a $C^{k}$ topological contraction of $E$.

Notice that if the map $\varphi$ appearing in (E4) is also globally Lipschitz, we could argue as in Lemma 4.2, writing $f=T \circ\left(\mathrm{id}+f_{0}\right)$ and then using $\varphi$ to extend $f_{0}$ to a $C^{k}$ map on $E$ with small Lipschitz norm. Without this assumption, a natural idea is to see id $+f_{0}$ as the time-one map obtained by integrating a time dependent small vector field $X$, and then use $\varphi$ to extend $X$. We therefore need the following easy:

LEMMA 4.8. Let $1 \leq k \leq \omega$, let $U \subset E$ be an open neighborhood of 0 , and let $g: U \rightarrow E$ be a $C^{k}$ map such that $g(0)=0$ and $D g(0)=I$. Then there exists a neighborhood $V \subset U$ of 0 and a $C^{k} \operatorname{map} X:[0,1] \times V \rightarrow E$ such that $X(t, 0)=0, D_{2} X(t, 0)=0$ for every $t \in[0,1]$, and the solution of the Cauchy problem

$$
\partial_{t} G(t, \xi)=X(t, G(t, \xi)), \quad G(0, \xi)=\xi,
$$

satisfies $G(1, \xi)=g(\xi)$ for every $\xi$ in some neighborhood of 0 .

Proof. The differential with respect to the second variable of the $C^{k}$ map

$$
G:[0,1] \times U \rightarrow E, \quad G(t, \xi)=\operatorname{tg}(\xi)+(1-t) \xi,
$$

namely $t D g(\xi)+(1-t) I$, is uniformly invertible for every $(t, \xi)$ in a neighborhood of $[0,1] \times\{0\}$. By the parametric inverse mapping theorem, there exist a neighborhood $V$ of 0 and a $C^{k}$ map $H:[0,1] \times V \rightarrow E$ such that

$$
H(t, G(t, \xi))=\xi \quad \forall(t, \xi) \in[0,1] \times V .
$$

Setting

$$
X(t, \eta)=g(H(t, \eta))-H(t, \eta),
$$

we conclude that $X$ has the desired properties. 
We can now prove Proposition 4.7.

Proof of Proposition 4.7. Let $T=D f(0)$ and let $|\cdot|$ be an adapted norm for $T$, so that $T$ becomes a contraction. Consider the diffeomorphism $g=$ $T^{-1} f$, whose differential at 0 is the identity operator. Let $X \in C^{k}([0,1] \times V, E)$ and $G$ be as in Lemma 4.8. By assumption, there is a $C^{k}$ map $\varphi: E \rightarrow E$ whose image is contained in $B_{r_{0}}(0)$, which coincides with the identity map on $B_{s_{0}}(0)$, for some $0<s_{0}<r_{0}<+\infty$. Since $X(t, 0)=0$ and $D_{2} X(t, 0)=0$ for every $t \in[0,1]$, we can find $r>0$ such that

$$
|X(t, \xi)| \leq \varepsilon|\xi| \quad \forall(t, \xi) \in[0,1] \times B_{r}(0),
$$

where $\varepsilon>0$ is so small that $e^{\varepsilon r_{0} / s_{0}}\|T\|<1$. The $C^{k}$ map

$$
\psi(\xi)=\frac{r}{r_{0}} \varphi\left(\frac{r_{0}}{r} \xi\right)
$$

takes values in $B_{r}(0)$, and coincides with the identity mapping on $B_{s}(0)$, with $s=r s_{0} / r_{0}$. The time dependent $C^{k}$ vector field

$$
\widetilde{X}:[0,1] \times E \rightarrow E, \quad \widetilde{X}(t, \xi)=X(t, \psi(\xi)),
$$

coincides with $X$ on $[0,1] \times B_{s}(0)$, so by $(5),|\widetilde{X}(t, \xi)| \leq \varepsilon|\xi|$ there. On the other hand, if $|\xi| \geq s$,

$$
|\widetilde{X}(t, \xi)|=|X(t, \psi(\xi))| \leq \varepsilon|\psi(\xi)| \leq \varepsilon r \leq \varepsilon \frac{r}{s}|\xi|=\varepsilon \frac{r_{0}}{s_{0}}|\xi| .
$$

We conclude that the above estimate holds for every $(t, \xi) \in[0,1] \times E$. Therefore, the solution $\widetilde{G}$ of the Cauchy problem

$$
\partial_{t} \widetilde{G}(t, \xi)=\widetilde{X}(t, \widetilde{G}(t, \xi)), \quad \widetilde{G}(0, \xi)=\xi,
$$

is defined for every $(t, \xi) \in[0,1] \times E$, coincides with $G$ in a neighborhood of $[0,1] \times\{0\}$, and by $(6)$ it satisfies

$$
|\widetilde{G}(t, \xi)| \leq e^{\varepsilon r_{0} t / s_{0}}|\xi| \quad \forall(t, \xi) \in[0,1] \times E .
$$

Since $G(1, \xi)=g(\xi)$, the global $C^{k}$ diffeomorphism

$$
\widetilde{g}: E \rightarrow E, \quad \widetilde{g}(\xi)=\widetilde{G}(1, \xi),
$$

coincides with $g$ in a neighborhood of 0 . Then the $C^{k}$ diffeomorphism $\widetilde{f}=T \widetilde{g}$ coincides with $f$ in a neighborhood of 0 . Finally, by (7),

$$
|\widetilde{f}(\xi)| \leq\|T\||\widetilde{g}(\xi)| \leq\|T\| e^{\varepsilon r_{0} / s_{0}}|\xi|,
$$

and the fact that $e^{\varepsilon r_{0} / s_{0}}\|T\|<1$ implies that the hyperbolic fixed point 0 is a global attractor.

The smooth and analytic cases. It remains to prove part (ii) of Theorem B. Let us start by examining some consequences of assumption (E5) (here by Taylor polynomial we mean Taylor polynomial at 0 ): 
LEMmA 4.9. Let $k=\infty$ or $k=\omega$. The following facts are equivalent:

(i) there exists a bounded map $\varphi \in C^{k}(E, E)$ such that $\varphi(0)=0$ and $D \varphi(0)=I$

(ii) every polynomial map $p: E \rightarrow E$ with $\operatorname{deg} p \leq n$ is the Taylor polynomial of order $n$ of some bounded map $\psi \in C^{k}(E, E)$;

(iii) if $\varepsilon>0, K$ is a compact manifold of class $C^{h}, 0 \leq h \leq \omega, U$ is an open neighborhood of 0 in $E, F$ is a Banach space, and $\phi: K \times U \rightarrow$ $F$ is a $C^{h}$ map such that for every $x \in K$ the $\operatorname{map} \phi(x, \cdot): E \rightarrow F$ is of class $C^{k}$ and $\phi(x, 0)=0$, then there exists a map $\psi: K \times E \rightarrow F$ with the same regularity such that $\sup |\psi|<\varepsilon$ and for every $x \in K$ the Taylor polynomials of order $n$ of $\phi(x, \cdot)$ and of $\psi(x, \cdot)$ coincide.

Proof. Statement (i) is a particular case of (ii): take $p(x)=x$ and $n=1$. Statement (ii) is a particular case of (iii): take $K$ a singleton, $\phi=p-p(0)$. So it is enough to prove that (i) implies (iii). Given $\varphi$ satisfying (i), it is easy to construct a bounded map $\varphi_{n} \in C^{k}(E, E)$ such that $\varphi_{n}(\xi)=\xi+o\left(|\xi|^{n}\right)$ as $\xi \rightarrow 0$. Indeed, one may define $\varphi_{n}$ inductively as

$$
\left\{\begin{array}{l}
\varphi_{1}=\varphi \\
\varphi_{n+1}(\xi)=\varphi_{n}\left(\xi-\frac{1}{(n+1) !} D^{n+1} \varphi_{n}(0) \xi^{n+1}\right) .
\end{array}\right.
$$

Let $\phi: K \times U \rightarrow F$ be the map appearing in (iii). By replacing $\varphi(\xi)$ by $\lambda \varphi(\xi / \lambda)$ in the above construction, we may assume that the image of $\varphi$ (hence also of $\varphi_{n}$ ) is contained in a neighborhood $V \subset U$ of 0 which is so small that $\sup _{K \times V}|\phi|<\varepsilon$ (such a neighborhood exists because $\phi$ is continuous, and it vanishes on the compact set $K \times\{0\})$. Then the map

$$
\psi(x, \xi)=\phi\left(x, \varphi_{n}(\xi)\right)
$$

has the required properties.

The proof of Proposition 4.11 below relies on the following classical conjugacy result (see for instance [CFdlL03a]):

ThEOREM 4.10. Let $k=\infty$ or $k=\omega$. Let $U \subset E$ be an open neighborhood of 0 , and let $f: U \rightarrow f(U) \subset E$ be a $C^{k}$ map with $f(0)=0$, and $D f(0)=T$ an isomorphism with spectral radius $\varrho(T)<1$. Then $f$ is $C^{k}$ locally conjugate to its Taylor polynomial of order $n$, provided that $n$ is so large that $\varrho\left(T^{-1}\right) \varrho(T)^{n+1}<1$.

Theorem B(ii) is a consequence of Corollary 4.6 and of the following:

Proposition 4.11. Let $k=\infty$ or $k=\omega$, and assume that the Banach space $E$ satisfies condition (E5). Let $U \subset E$ be an open neighborhood of 0 , and let $f: U \rightarrow f(U) \subset E$ be a $C^{k}$ diffeomorphism with hyperbolic fixed point 0 which is a local attractor. Then the germ of $f$ at 0 extends up to conjugacy to a $C^{k}$ topological contraction of $E$. 
Proof. Let $T=D f(0)$ and let $|\cdot|$ be an adapted norm for $T$, so that $T$ becomes a contraction. Consider the diffeomorphism $g:=T^{-1} f$, whose differential at 0 is the identity operator. Let $X \in C^{k}([0,1] \times V, E)$ and $G$ be as in Lemma 4.8: $G$ is the map obtained by integrating the time dependent vector field $X$, and $G(1, \cdot)=g$ in a neighborhood of 0 .

Consider the $C^{k}$ map

$$
\phi:[0,1] \times V \rightarrow \mathcal{L}(E), \quad \phi(t, \xi)=D_{2} X(t, \xi) .
$$

Fix $n \in \mathbb{N}$ so large that

$$
\varrho\left(T^{-1}\right) \varrho(T)^{n+1}<1,
$$

and $\varepsilon>0$ so small that $e^{\varepsilon}\|T\|<1$. Since $\phi(t, 0)=D_{2} X(t, 0)=0$, by Lemma 4.9 there exists a $C^{k}$ map $\psi:[0,1] \times E \rightarrow L(E)$ such that

$$
\sup _{(t, \xi) \in[0,1] \times E}\|\psi(t, \xi)\|<\varepsilon
$$

and for every $t \in[0,1]$ the Taylor polynomials of order $n-1$ of $\phi(t, \cdot)$ and $\psi(t, \cdot)$ coincide. Equivalently,

$$
\psi(t, \xi)-\phi(t, \xi)=o\left(|\xi|^{n-1}\right) \quad \text { as } \xi \rightarrow 0,
$$

uniformly in $t \in[0,1]$. Consider the globally defined time dependent vector field of class $C^{k}$,

$$
\widetilde{X}:[0,1] \times E \rightarrow E, \quad \widetilde{X}(t, \xi)=\int_{0}^{1} \psi(t, s \xi) \xi d s .
$$

By (9),

$$
|\widetilde{X}(t, \xi)| \leq \int_{0}^{1}\|\psi(t, s \xi)\||\xi| d s \leq \varepsilon|\xi| \quad \forall(t, \xi) \in[0,1] \times E .
$$

Since

$$
X(t, \xi)=\int_{0}^{1} \frac{d}{d s} X(t, s \xi) d s=\int_{0}^{1} D_{2} X(t, s \xi) \xi d s=\int_{0}^{1} \phi(t, s \xi) \xi d s
$$

by (10) we have

$$
\widetilde{X}(t, \xi)-X(t, \xi)=\int_{0}^{1}(\psi(t, s \xi)-\phi(t, s \xi)) \xi d s=o\left(|\xi|^{n}\right) \quad \text { as } \xi \rightarrow 0,
$$

uniformly in $t \in[0,1]$. Therefore, $X(t, \cdot)$ and $\widetilde{X}(t, \cdot)$ have the same Taylor polynomial of order $n$. An easy induction argument then shows that $G(t, \cdot)$ and the solution $\widetilde{G}(t, \cdot)$ of the Cauchy problem

$$
\partial_{t} \widetilde{G}(t, \xi)=\widetilde{X}(t, \widetilde{G}(t, \xi)), \quad \widetilde{G}(0, \xi)=0,
$$

have the same Taylor polynomial of order $n$, for every $t \in[0,1]$. In particular, $g(\xi)=G(1, \xi)$ and $\widetilde{g}(\xi):=\widetilde{G}(1, \xi)$ have the same Taylor polynomial of 
order $n$. The same happens for $f=T \circ g$ and $\widetilde{f}:=T \circ \widetilde{g}$, so by (8) Theorem 4.10 implies that $f$ and $\widetilde{f}$ are $C^{k}$ locally conjugate at 0 .

Since $\widetilde{X}$ has linear growth by (11), the map $G$ is well defined on $[0,1] \times E$, and $G(t, \cdot)$ is a $C^{k}$ diffeomorphism of $E$ onto $E$ for every $t \in[0,1]$. By (11),

$$
|\widetilde{f}(\xi)| \leq\|T\||\widetilde{g}(\xi)| \leq\|T\| e^{\varepsilon}|\xi|,
$$

so the fact that $e^{\varepsilon}\|T\|<1$ implies that $\tilde{f}$ is a topological contraction of $E$, concluding the proof.

\section{References}

[AM04] A. Abbondandolo and P. Majer, Lectures on the Morse complex for infinite dimensional manifolds, in: Morse Theoretic Methods in Nonlinear Analysis and in Symplectic Topology, NATO Sci. Ser. II Math. Phys. Chem., P. Biran et al. (eds.), 2006, 1-74.

[Atk01] C. J. Atkin, Extension of smooth functions in infinite dimensions, I: unions of convex sets, Studia Math. 146 (2001), 201-226.

[CFdlL03a] X. Cabré, E. Fontich, and R. de la Llave, The parametrization method for invariant manifolds I: manifolds associated to non-resonant subspaces, Indiana Univ. Math. J. 52 (2003), 283-328.

[CFdlL03b] -, -, 一, The parametrization method for invariant manifolds II: regularity with respect to parameters, ibid., 329-360.

[FHY83] A. Fathi, M. R. Herman and J.-C. Yoccoz, A proof of Pesin's stable manifold theorem, in: Geometric Dynamics (Rio de Janeiro, 1981), J. Palis (ed.), Springer, 1983, 177-215.

[HPS77] M. W. Hirsch, C. C. Pugh, and M. Shub, Invariant Manifolds, Lecture Notes in Math. 583, Springer, Berlin, 1977.

[Irw70] M. C. Irwin, On the stable manifold theorem, Bull. London Math. Soc. 2 (1970), 196-198.

[Irw80] - A new proof of the pseudostable manifold theorem, J. London Math. Soc. 21 (1980), 557-566.

[Lan99] S. Lang, Fundamentals of Differential Geometry, Springer, Berlin, 1999.

[Shu87] M. Shub, Global Stability of Dynamical Systems, Springer, New York, 1987.

[Wel76] J. C. Wells, Invariant manifolds of non-linear operators, Pacific J. Math. 62 (1976), 285-293.

[Wig94] S. Wiggins, Normally Hyperbolic Invariant Manifolds in Dynamical Systems, Springer, New York, 1994.

Dipartimento di Matematica

Università di Pisa

Largo Bruno Pontecorvo 5

56127 Pisa, Italy

E-mail: abbondandolo@dm.unipi.it

Web: http://www.dm.unipi.it/ abbondandolo/ 